\title{
Another Glance at the Alpern-Schneider Characterization of Safety and Liveness in Concurrent Executions
}

\author{
H.Peter Gumm
}

\begin{abstract}
In order to derive a result such as the Alpern-Schneider theorem characterizing safety and liveness properties of concurrent program executions, it is shown that all that is needed is a $\vee$-preserving map $\varphi$ between complete Boolean algebras. Every property becomes a conjunction of a safety and a liveness property and safety properties can be characterized by sets of configurations that are to be "avoided".

Aside from the original result of B. Alpern and F.B. Schneider we also provide a new application by considering transition systems with a UNITY-style logic. Safety properties are characterized by a set of forbidden pairs of successive states and progress properties are those allowing all possible state-successor pairs. Every property of a transition system is shown to be a conjunction of a safety and a progress property.
\end{abstract}

Keywords : Safety, Liveness, Progress, Concurrency, Semantics, UNITY.

\section{Introduction}

It is well known [4] that temporal properties of concurrent programs in general display two aspects: A safety aspect expressing that "nothing will go wrong" and a liveness aspect asserting that "something desired will eventually happen". This imprecise observation was first made precise in the work of B. Alpern and F.B. Schneider [1] where convincing formal definitions of liveness and safety were given and it was shown that every property is a conjunction of a safety and a liveness property.

M. Rem in [5] shed new light on the above mentioned theorem, mainly by defining an equivalence relation on properties so that safety properties are in 1-1 correspondence with the equivalence classes and progress properties are precisely the members of the equivalence class to which True belongs. The topological closure operator (the closure of $a$ is written $\bar{a}$ ) used in [1] was given a concrete definition, so safety properties could be understood as those properties $P$ with $\bar{P}=P$. 
In this article we take up the the theme again, initially placing it in a more abstract setting. The theorem becomes a result about a $V$-preserving map between two $\vee$-complete Boolean algebras. The application of the theorem to the AlpernSchneider case is immediate, but we also present a second application which yields a new Alpern-Schneider type result suitable for transition systems whose behaviours are described in terms of properties of the (one-step) transitions: Replacing "liveness" by "progress", we obtain that every property is a conjunction of a progress property and a safety property.

In our second theorem we show that safety properties are precisely the properties definable by a set of "configurations" that are to be avoided. Safety properties are closed under finite conjunction.

In our proofs we get by without needing to show $\bar{P} \vee \bar{Q}=\overline{P \vee Q}$ as is done in [5]. This property is in fact false in our second application.

\section{Main Results}

Let $B_{1}$ and $B_{2}$ be $\vee$-complete ${ }^{1}$ Boolean algebras and $\varphi: B_{1} \longrightarrow B_{2}$ a $\vee$ preserving map. Let $\sim$ be the kernel of $\varphi$, i.e.

$$
a \sim b \Leftrightarrow \varphi(a)=\varphi(b)
$$

Define the closure $\bar{a}$ of an element $a \in B_{1}$ as

$$
\bar{a}=\bigvee\left\{x \in B_{1} \mid x \sim a\right\} .
$$

For elements $e \in B_{1}$ define :

$$
\begin{array}{ll}
e \text { is a safety element } & \Leftrightarrow \bar{e}=e \\
e \text { is a liveness element } & \Leftrightarrow \bar{e}=1 .
\end{array}
$$

Theorem 1 Every element $e \in B_{1}$ is a conjunction of a safety and a liveness element.

Theorem 2 An element $e \in B_{1}$ is a safety element if and only if there exists $u \in B_{2}$ so that

$$
e=\bigvee\left\{x \in B_{1} \mid \varphi(x) \wedge u=0\right\} \quad ;
$$

$e$ is actually the largest element whose $\varphi$-image avoids $u$.

A careful analysis of the proofs shows that we only require that $B_{1}$ is a $V$ complete and complemented modular lattice. $B_{2}$ must be a $\vee$-semilattice for theorem 1 and a complemented lattice for theorem 2 .

Our original proof of theorem 2 assumed that $\varphi$ was onto. M. Müller noticed that this assumption was not necessary. He also contributed the following corollary :

Corollary 1 Safety elements are closed under finite conjunctions.

\footnotetext{
${ }^{1}$ infinite suprema exist
} 


\section{Applications}

Application 1 For a set $A$ (of states) let $\preceq$ denote the prefix relation between elements of $A^{*}$ and elements of $A^{\omega}$. For $P \subseteq A^{\omega}$ let

$$
\varphi(P)=\left\{x \in A^{*} \mid \exists \alpha \in P: x \preceq \alpha\right\} .
$$

In this setup theorem 1 yields the Alpern-Schneider theorem. $A$ is a set of possible states of a computation. $A^{\omega}$ is the set of all possible executions and $A^{*}$ the set of all finite (or partial) executions. $B_{1}$ and $B_{2}$ are the powersets of $A^{\omega}$ and $A^{*}$. The fact that $\varphi$ is $\vee$-preserving is trivial to check.

Elements of $B_{1}$ are called properties. A liveness property then is a property that admits every possible partial execution, and a safety property is a property that is maximal with respect to a given set of allowed partial executions.

Theorem 2 states in this connection that safety properties $E$ are those properties that can be given by a set $U$ of disallowed partial executions.

Application 2 For a set $A$ ( of states) let $\asymp$ denote the following relation between $A^{2}$ and $A^{\omega}$ :

$$
(x, y) \asymp \alpha \Leftrightarrow \exists i \in \omega:(x, y)=\left(\alpha_{i}, \alpha_{i+1}\right) .
$$

For $P \subseteq A^{\omega}$ let

$$
\varphi(P)=\left\{(x, y) \in A^{2} \mid \exists \alpha \in P:(x, y) \asymp \alpha\right\} .
$$

Note that properties allowing all possible one-step transitions are usually called progress properties. In our notation : $P \subseteq A^{\omega}$ is a progress property iff $\forall x, y \in A \exists \tau \in P:(x, y) \asymp \tau$.

In the present setting, with $B_{1}$ and $B_{2}$ the powersets of $A^{\omega}$ and $A^{2}$, and the above $\vee$-preserving map $\varphi$, the liveness elements given by the abstract theory correspond to the progress properties as defined above. The safety elements correspond to those properties that can be defined by a set $F$ of forbidden state-successor pairs. This, however, amounts to forbidding all finite prefixes containing a pair from F, so every safety element is also a safety property in the usual sense. Therefore we get :

Theorem 3 Every property is a conjunction of a safety property and a progress property.

This application seems particularly relevant for transition systems whose logical calculus is based on properties of single transitions. In UNITY, [3] for instance, properties are based on Hoare-triples $\{p\} s\{q\}$ where $p$ and $q$ are first order properties and $s$ is a transition, given by a multiple conditional assignment.

Safety properties are derived from the basic temporal property unless, which is defined by quantifying over all statements $s$ in a program $P$ :

$$
p \text { unless } q \Leftrightarrow \forall s \in P:\{p \wedge \neg q\} s\{p \vee q\} \text {. }
$$


An execution $\alpha$ therefore satisfies $p$ unless $q$ if and only if there is no pair $(x, y) \asymp \alpha$ with $x \models p \wedge \neg q$ and $y \models \neg p \wedge \neg q$.

A similar remark applies to the derived unary safety property invariant, given by :

$$
\text { invariant } q \Leftrightarrow q \text { unless False. }
$$

\section{Proofs}

Given a $\vee$-preserving map $\varphi$ between $\vee$-complete Boolean algebras $B_{1}$ and $B_{2}$ as requested, together with the definitions

$$
a \sim b \Leftrightarrow \varphi(a)=\varphi(b),
$$

and

$$
\bar{a}=\bigvee\left\{x \in B_{1} \mid x \sim a\right\},
$$

we first show the following properties:

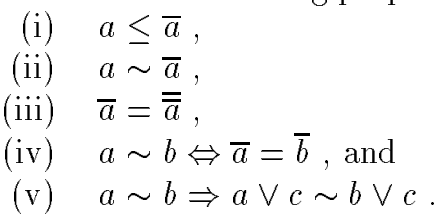

From the definition of $\bar{a}$ and the reflexivity of $\sim$, (i) is immediate. For (ii) we calculate :

$$
\begin{aligned}
\varphi(\bar{a}) & =\varphi\left(\bigvee\left\{x \in B_{1} \mid x \sim a\right\}\right) \\
& =\bigvee\left\{\varphi(x) \mid x \in B_{1}, x \sim a\right\} \\
& =\bigvee\left\{\varphi(x) \mid x \in B_{1}, \varphi(x)=\varphi(a)\right\} \\
& =\bigvee\{\varphi(a)\} \\
& =\varphi(a) .
\end{aligned}
$$

Hence $a \sim \bar{a}$, and

$$
\begin{aligned}
\overline{\bar{a}} & =\bigvee\left\{x \in B_{1} \mid \varphi(x)=\varphi(\bar{a})\right\} \\
& =\bigvee\left\{x \in B_{1} \mid \varphi(x)=\varphi(a)\right\} \\
& =\bar{a} .
\end{aligned}
$$

If $a \sim b$ then $\bar{a}=\bar{b}$ from the definition of the closure operator. Conversely, if $\bar{a}=\bar{b}$ then $a \sim \bar{a}=\bar{b} \sim b$ using (ii). Property (v) follows from the fact that $\varphi$ preserves $\vee$ : If $\varphi(a)=\varphi(b)$ then

$$
\begin{aligned}
\varphi(a \vee c) & =\varphi(a) \vee \varphi(c) \\
& =\varphi(b) \vee \varphi(c) \\
& =\varphi(b \vee c) .
\end{aligned}
$$


To show Theorem 1 we now use the same decomposition as [1] and [5]: Given any $e \in B_{1}$, then using (i) and the modular law [2] :

$$
\begin{aligned}
\bar{e} \wedge(e \vee \neg \bar{e}) & =e \vee(\bar{e} \wedge \neg \bar{e}) \\
& =e .
\end{aligned}
$$

$\bar{e}$ is a safety element from (iii), and $e \sim \bar{e}$ from (ii), so from (v) : $e \vee \neg \bar{e} \sim$ $\bar{e} \vee \neg \bar{e}=1_{B_{1}}$, is a progress element.

Proof of theorem 2: We need two further properties for arbitrary $a \in B_{1}$ and $u \in B_{2}$ :

(vi) $\bar{a}=\bigvee\left\{x \in B_{1} \mid \varphi(x) \leq \varphi(a)\right\}$, and

(vii) $a=\bigvee\left\{x \in B_{1} \mid \varphi(x) \leq u\right\} \Rightarrow a=\bar{a}$.

For (vi) we show that $\bigvee\left\{x \in B_{1} \mid \varphi(x)=\varphi(a)\right\}=\bigvee\left\{x \in B_{1} \mid \varphi(x) \leq \varphi(a)\right\}$. Containment of the left hand side in the right hand side is obvious, since less elements are involved in the join. For the inverse containment, let $\varphi(x) \leq \varphi(a)$, then $\varphi(x \vee a)=\varphi(x) \vee \varphi(a)=\varphi(a)$, so $x \leq(x \vee a) \leq \bar{a}$.

Assuming the hypothesis of (vii), we need to conclude $\bar{a} \leq a$. From the description of $\bar{a}$ in (vi), it is enough to derive the implication $\varphi(x) \leq \varphi(a) \Rightarrow$ $x \leq a$. But from the definition of $a$ in the hypothesis of (vii),

$$
\begin{aligned}
\varphi(x) & \leq \varphi(a) \\
& =\varphi(\bigvee\{x \mid \varphi(x) \leq u\}) \\
& =\bigvee\{\varphi(x) \mid \varphi(x) \leq u\} \\
& \leq u,
\end{aligned}
$$

hence $x \leq a$.

From (vi) and the fact that $y \leq z$ implies that $y \wedge \neg z=0$ we now obtain the first direction of theorem 2, namely

$$
\bar{e}=\bigvee\left\{x \in B_{1} \mid \varphi(x) \wedge \neg \varphi(e)=0\right\}
$$

For the other direction suppose $e=\bigvee\left\{x \in B_{1} \mid \varphi(x) \wedge u=0\right\}$, which means that $e=\bigvee\{x \mid \varphi(x) \leq \neg u\}$ for some $u \in B_{2}$. By (vii) $e=\bar{e}$, so $e$ is a safety element.

To show that $e$ is actually the largest element $x$ with $\varphi(x) \wedge u=0$ we need only see that $\varphi(e) \wedge u=0$. But

$$
\begin{aligned}
\varphi(e) & =\varphi\left(\bigvee\left\{x \in B_{1} \mid \varphi(x) \wedge u=0\right\}\right) \\
& =\bigvee\left\{\varphi(x) \mid x \in B_{1}, \varphi(x) \wedge u=0\right\} \\
& \leq \bigvee\left\{v \in B_{2} \mid v \wedge u=0\right\} \\
& =\neg u
\end{aligned}
$$


Proof of the Corollary: $e_{1}$ and $e_{2}$ are safety elements, so by theorem 2, $e_{1}=\bigvee\left\{x \in B_{1} \mid \varphi(x) \leq u_{1}\right\}$ and $e_{2}=\bigvee\left\{x \in B_{1} \mid \varphi(x) \leq u_{2}\right\}$. In particular, $\varphi\left(e_{1}\right) \leq u_{1}$ and $\varphi\left(e_{2}\right) \leq u_{2}$. Since $\varphi$ is monotone, $\varphi\left(e_{1} \wedge e_{2}\right) \leq \varphi\left(e_{1}\right) \wedge \varphi\left(e_{2}\right)$, hence $\varphi\left(e_{1} \wedge e_{2}\right) \leq u_{1} \wedge u_{2}$ and $e_{1} \wedge e_{2} \leq \bigvee\left\{x \in B_{1} \mid \varphi(x) \leq u_{1} \wedge u_{2}\right\}$. Conversely, if $\varphi(x) \leq u_{1} \wedge u_{2}$ then $x \leq e_{1} \wedge e_{2}$, so $e_{1} \wedge e_{2}=\bigvee\left\{x \in B_{1} \mid \varphi(x) \leq u_{1} \wedge u_{2}\right\}$, and by theorem 2 this is a safety element.

\section{Conclusion}

We have worked out the mathematical setup that is required to derive a result in the style of the Alpern-Schneider theorem characterizing safety and liveness. We have arrived at a more general result that directly yields the mentioned theorem, but that also broadens its application to transition systems whose temporal properties are given by properties of (one-step) transitions. In that case safety properties are those properties definable by a set of allowable state pairs $\left(a_{t}, a_{t+1}\right)$ or, equivalently, by a set of such forbidden pairs. Progress properties are properties allowing each such possible pair, and every property is a conjunction of a safety property and a progress property.

\section{References}

[1] B. Alpern and F.B. Schneider. Defining Liveness. Information Processing Letters, 21 (1985), 181-185.

[2] G. Birkhoff. Lattice theory. A.M.S. Colloquium Publ., Providence, R.I. 1967.

[3] K.M. Chandy, J. Misra. Parallel Program Design. Addison Wesley, 1988.

[4] L. Lamport. Proving the correctness of multiprocess programs. IEEE Trans. on Software Engr. SE-3, 2(March), 125-143.

[5] M. Rem . A Personal Perspective of the Alpern-Schneider Characterization of Safety and Liveness, in W.H.J. Feijen, et al. Beauty is Our Business, Springer Verlag, 1990, 365-372. 